\title{
A comprehensive study of microbiological profile, risk factors and antibiotic sensitivity pattern of catheter associated urinary tract infection in a teaching hospital of Gujarat
}

\author{
Dipak Motilal Panjwani ${ }^{1,2, *}$, Sucheta Jitendra Lakhani ${ }^{3}$, Sanjay Jayantilal Mehta ${ }^{2}$, Kunjan Madhukar Kikani ${ }^{2}$, \\ Khushi Shyam Shah ${ }^{2}$ \\ ${ }^{1}$ Department of Microbiology, Sumandeep Vidyapeeth Deemed to be University, Vadodara, India. \\ ${ }^{2}$ Department of Microbiology, C. U. Shah Medical College, Surendranagar, India. \\ ${ }^{3}$ Department of Microbiology, SBKS MI \& RC, Sumandeep Vidyapeeth Deemed to be University, Vadodara, India.
}

\section{ARTICLE INFO \\ Article history: \\ Received on: March 09, 2021 \\ Accepted on: June 26, 2021 \\ Available online: September 01, 2021}

Key words:

Hospital-acquired infection, CAUTI, CA-ASB, antibiotic sensitivity patterns

\begin{abstract}
The current study was carried out to find the microbiological profile along with the risk factors and antibiotic sensitivity patterns of catheter-associated urinary tract infection in a teaching hospital. All the catheterized patients satisfying the criteria of catheter-associated urinary infection (CAUTI) and catheter-associated asymptomatic bacteriuria (CA-ASB) were included in the study. Urine samples received from CAUTI patients were followed-up further. Samples were collected and inoculated on MacConkey's agar and Blood agar. Identification and antibiotic sensitivity patterns of bacterial isolates were carried out by automated system, i.e., Vitek-2, as per standard protocols. Duration of catheterization and various risk factors were also recorded in detail. Out of 247 urine samples of hospital acquired infection, 50 samples satisfied the criteria of CAUTI. The most common organism isolated was Escherichia coli (38\%), followed by Pseudomonas sp. (24\%) and Klebsiella sp. (24\%). Maximum isolates were from the medicine ward (40\%). Gram-negative isolates showed dominance over the Gram-positive isolates. Such increasing figures of CAUTI incidents pose a great challenge to the hospital management and threat to the patients with underlying illness. With strict adherence to the catheter care of patients and use of infection control practices, we can significantly decline the rate of developing CAUTI.
\end{abstract}

\section{INTRODUCTION}

Hospital-acquired urinary tract infection (UTI) is one of the most commonly encountered type of hospital-acquired infections. Catheter-associated urinary infection (CAUTI) needs prompt diagnosis and treatment; otherwise it may lead to chronic renal complications. These infections are difficult to treat due to higher resistance strains of nosocomial bacteria.

According to the Infectious Disease Society of America, if the patient has an indwelling urinary catheter for $>48$ hours along with at least one sign or symptom like fever $>38^{\circ} \mathrm{C}$, suprapubic

*Corresponding Author

Dipak Motilal Panjwani, Ph.D. Scholar, Department of Microbiology,

Sumandeep Vidyapeeth Deemed to be University, Vadodara, India.

E-mail: dipakpanjwani98@gmail.com tenderness, or costovertebral angle pain, it is considered as CAUTI. On the other side, if the patient has a catheter for $>48$ hours without having such symptoms, it is categorized as catheter-associated asymptomatic bacteriuria (CA-ASB). This CA-ASB event is most commonly associated after the patients have had catheter, but not all CA-ASB events are transformed into CAUTI [1].

Hospital-acquired UTI is reported in a very wide range from $1 \%-5 \%$ to $100 \%$ for patients who had $>48$ hours of indwelling urinary catheter. This wide range of UTIs is dependent on the type of urinary catheter, number of catheter days of patient, catheter care of patients, immunity of host, and infection control practices of hospital [2-4]. 


\section{MATERIALS AND METHODS}

\subsection{Study Design}

This is an observational clinical study carried out in a teaching hospital during January 2018 to June 2020.

\subsubsection{Study site}

The study site comprised all the wards and Intensive care unit Intensive care units (ICUs) of C. U. Shah Medical College and Hospital, Surendranagar, Gujarat, India.

\subsubsection{Patients}

All the patients with indwelling urinary catheter in the last 48 hours without any previous history /symptoms of UTI were included in the study.

\subsubsection{Inclusion criteria}

All catheterized patients who are admitted in the hospital and acquired UTI, which was not present or incubating at the time of admission, were included in the study. UTIs occurring more than 48 hours after admission are considered as nosocomial. Patients having one of the symptoms like fever, suprapubic tenderness, or costovertebral angle pain are considered having CAUTI and were included in study.

\subsubsection{Sample collection}

Urine sample was collected from patients having indwelling urinary catheters by clamping of tube to allow freshly voided urine. Before collecting the sample, tubing was cleaned with a $70 \%$ alcohol impregnated swab and the sample was collected via needle and syringe and transferred into a sterile container.

\subsubsection{Identification of isolates}

Samples were collected and processed for isolation and identification. Urine samples were inoculated on MacConkey's agar and Blood agar. Primary identification was carried out by Gram-staining and colony characteristics. Bacterial isolates were further identified with the help of the Vitek-2 automated instrument as per standard guidelines $[5,6]$.

\subsubsection{Antibiotic sensitivity test}

Isolated colonies were used for antibiotic sensitivity tests along with the detection of an extended spectrum $\beta$-lactamase (ESBL) and metallo- $\beta$-lactamase enzymes (MBL). Vancomycin-resistant strains of Enterococcus sp. were also identified. This test was carried out by the Vitek-2 automated instrument using minimum inhibitory concentration detection as per Clinical and Laboratory Standards Institute guideline 2018 [7].

\subsubsection{Ethical consideration}

The study was approved by the Institutional Ethics Committee (Human Research), C. U. Shah Medical College, Surendranagar, Gujarat, India.

\section{RESULT AND DISCUSSION}

Samples collected from various wards and ICUs were studied for the period of January 2018 to June 2020. During the study period, a total of 1,000 patients were identified as hospital acquired infection (HAI). Among these HAI patients, we have differentiated 247 hospital-acquired UTIs. From these 247 patients, 123 patients had indwelling urinary catheters. Out of 123 patients, $73(59 \%)$ catheterized patients showed no symptoms but there was growth of uropathogen on culture and hence were considered CA-ASB, while $50(41 \%)$ catheterized patients showed symptoms and hence considered CAUTI. The current study followed CA-ASB up to the identification of colonizing organisms, but has not followed-up with them further.

According to the ward-wise distribution of CAUTI isolates, the highest isolates were reported from the medicine ward $(40 \%)$, followed by surgery ward $(32 \%)$, while the least isolates were from the obstetrics and gynecology ward (2\%). Medicine ICU reported $14 \%$ of isolates and among these, the majority of the patients had various predisposing factors (Fig. 1)

The data of nosocomial pathogens from CAUTI patients revealed that Gram-negative isolates showed dominance over Grampositive isolates. The most common isolate encountered was

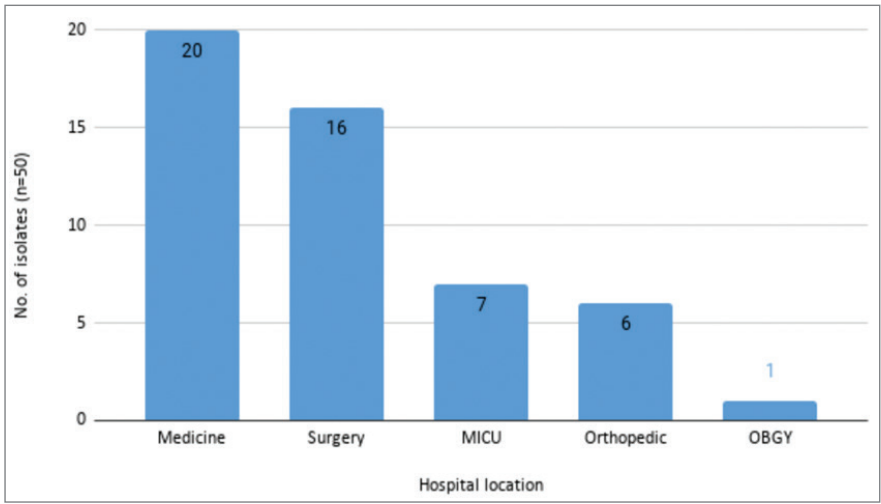

Figure 1. Ward wise distribution of CAUTI $(n=50)$. X-axis shows names of wards and ICUs and Y-axis shows number of CAUTI isolates $(n=50)$ recovered from these wards and ICUs. The maximum isolates were recovered from the medicine ward.

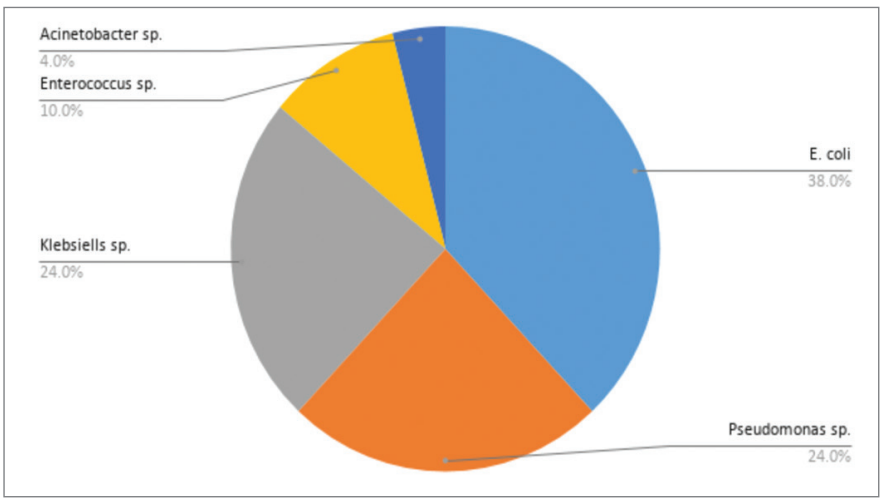

Figure 2. Organism-wise distribution of CAUTI $(n=50)$. The most commonly encountered organism was E. coli, followed by Pseudomonas sp. and Klebsiella sp. 
Escherichia coli (38\%), followed by Pseudomonas sp. (24\%) and Klebsiella sp. (24\%). In Gram-positive pathogens, the only organism isolated was Enterococcus sp. (10\%) (Fig. 2).

In the current study, we have noted that the organism profile of CA-ASB isolates was quite similar to CAUTI. CA-ASB isolates also showed a greater number $(85 \%)$ of Gram-negative isolates compared to Gram-positive isolates (15\%). Escherichia coli $(53 \%)$ was the most predominant pathogen in the case of CAASB, followed by Klebsiella sp. (21\%), Enterococcus sp. (11\%), and Pseudomonas sp. (10\%), respectively (Fig. 3).

We have also studied various risk factors and predisposing factors of CAUTI patients in detail. We have noted positive associations of age groups and the number of CAUTI patients. Maximum isolates were from patients who were older than 55 years age (58\%), followed by the $46-55$ years age group (18\%). The second important risk factor was the duration of catheterization of patients. Maximum incidences were encountered with patients who had catheterization for more than 48 hours and less than 7

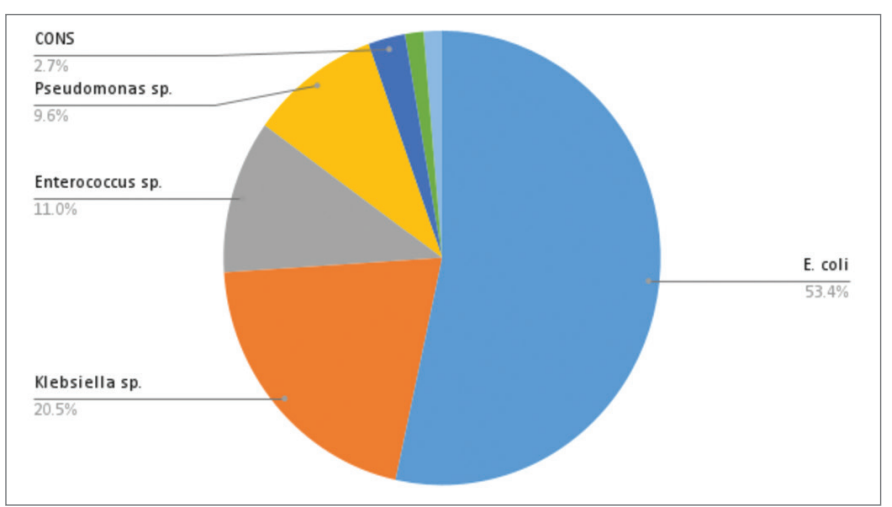

Figure 3. Organism-wise distribution of CA-ASB $(n=73)$. The most commonly encountered organism was E. coli, followed by Klebsiella sp. and Enterococcus sp.

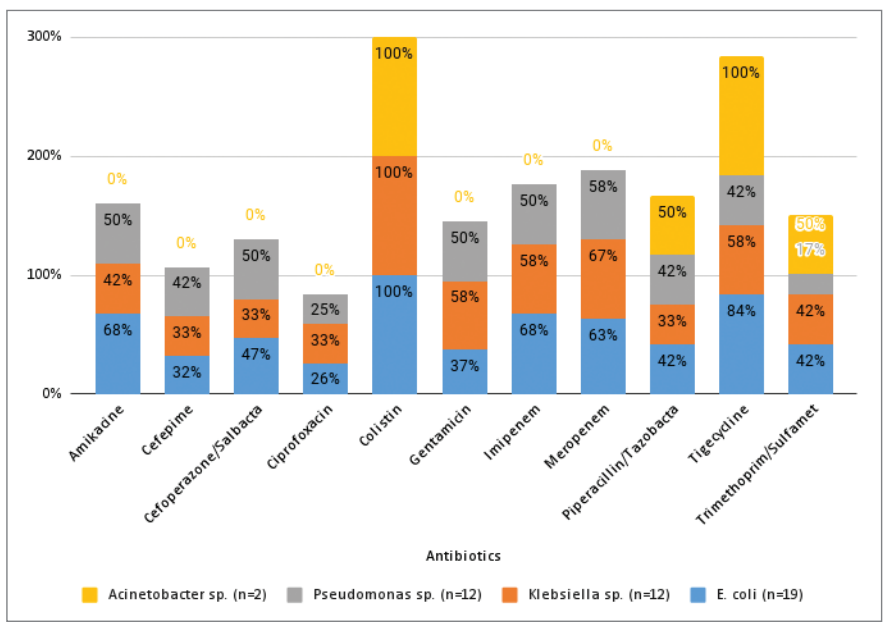

Figure 4. Antibiotic sensitivity patterns of various Gram-negative isolates. $\mathrm{X}$-axis shows percentage of isolates sensitive against antibiotics and $\mathrm{Y}$-axis shows commonly used antibiotics; the majority of organisms showed sensitivity against Colistin drug, while the least sensitive drug from the panel was Ciprofloxacin. days, while $20 \%$ of CAUTI patients had the catheter for more than 10 days. This could be possible due to the number of patients discharged after surgery or recovery from disease. Various predisposing factors like type 2 diabetes mellitus, hypertension, cerebrovascular accident, etc. were also studied during the study period, which are shown in Table 1.

According to the antibiotic sensitivity pattern of Gram-negative uropathogens, all the pathogens showed $100 \%$ sensitivity toward Colistin. Escherichia coli showed $84 \%$ sensitivity toward Tigecycline, while Klebsiella sp. showed $67 \%$ sensitivity toward Meropenem. Pseudomonas sp. showed maximum sensitivity to Meropenem (58\%). The least-sensitive drug from this panel was ciprofloxacin, showing only $21 \%$ sensitivity. We have noted that $20 \%$ of the pathogens were producing MBL and $18 \%$ of pathogens were producing ESBL enzymes. This could be a major challenge to treat CAUTI patients (Table 2; Fig. 4).

Antibiotic sensitivity of the only Gram-positive isolate, i.e., Enterococcus sp., was also studied. According to the sensitivity pattern, the most sensitive drugs of the panel were Teicoplanin and Linezolid (80\%), while Enterococcus sp. showed least sensitivity toward the fluoroquinolone group of drugs. We have detected $40 \%$ vancomycin-resistant Enterococcus strain during the study period (Table 3).

UTIs are the most common type of nosocomial infections. They account for $39 \%-40 \%$ of the total hospital acquired infections and this rate is even escalated when a patient acquires an indwelling urinary catheter. Prolonged use of catheter, poor catheter care, various predisposing factors, and lack of infection control policies may contribute to increasing cases of CAUTI. Almost $15 \%-20 \%$ of all the hospitalized patients required urinary catheterization

Table 1. Significant risk factor and predisposing factors of CAUTI patients.

\begin{tabular}{lcc}
$\begin{array}{l}\text { Significant risk factors and } \\
\text { predisposing factors }\end{array}$ & $\begin{array}{c}\text { CAUTI incidences } \\
(\boldsymbol{n}=\mathbf{5 0})\end{array}$ & Percentage \\
\hline Age & 29 & $58 \%$ \\
$>55$ years & 16 & $32 \%$ \\
$25-45$ years & 5 & $10 \%$ \\
$\quad<25$ years & & \\
Gender & 34 & $68 \%$ \\
Male & 16 & $32 \%$ \\
Female & & \\
Catheterization days & 37 & $74 \%$ \\
$>48$ hours & 3 & $6 \%$ \\
$>1$ week & 10 & $20 \%$ \\
$>10$ days & & \\
Predisposing factors & 5 & $10 \%$ \\
Diabetes Mellitus type - II & 5 & $10 \%$ \\
Hypertension & 5 & $10 \%$ \\
Cerebrovascular accident & 4 & $8 \%$ \\
Prostatomegaly & 2 & $4 \%$ \\
Other & & \\
\hline
\end{tabular}


Table 2. Antibiotic sensitivity pattern of Gram negative isolates.

\begin{tabular}{lcccc}
\multicolumn{1}{c}{ Antibiotics } & $\begin{array}{c}\boldsymbol{E} \text {. coli } \\
(\boldsymbol{n}=\mathbf{1 9 )}\end{array}$ & $\begin{array}{c}\text { Klebsiella sp. } \\
(\boldsymbol{n}=\mathbf{1 2})\end{array}$ & $\begin{array}{c}\text { Pseudomonas } \\
\mathbf{s p .}(\boldsymbol{n}=\mathbf{1 2})\end{array}$ & $\begin{array}{c}\text { Acinetobacter } \\
\text { sp. }(\boldsymbol{n}=\mathbf{2})\end{array}$ \\
Amikacin & $68 \%$ & $42 \%$ & $50 \%$ & $0 \%$ \\
Cefepime & $32 \%$ & $33 \%$ & $42 \%$ & $0 \%$ \\
Cefoperazone/Sulbactam & $47 \%$ & $33 \%$ & $50 \%$ & $0 \%$ \\
Ciprofloxacin & $26 \%$ & $33 \%$ & $25 \%$ & $0 \%$ \\
Colistin & $100 \%$ & $100 \%$ & $* *$ & $100 \%$ \\
Gentamicin & $37 \%$ & $58 \%$ & $50 \%$ & $0 \%$ \\
Imipenem & $68 \%$ & $58 \%$ & $50 \%$ & $0 \%$ \\
Meropenem & $63 \%$ & $67 \%$ & $58 \%$ & $0 \%$ \\
Piperacillin/Tazobactam & $42 \%$ & $33 \%$ & $42 \%$ & $50 \%$ \\
Tigecycline & $84 \%$ & $58 \%$ & $42 \%$ & $100 \%$ \\
Trimethoprim/Sulfamethoxazole & $42 \%$ & $42 \%$ & $17 \%$ & $50 \%$ \\
\hline
\end{tabular}

Table 3. Antibiotic sensitivity pattern of Gram positive isolates.

\begin{tabular}{|c|c|}
\hline Antibiotics & Enterococcus sp. $(n=5)$ \\
\hline Benzylpenicillin & $0 \%$ \\
\hline Ciprofloxacin & $0 \%$ \\
\hline Levofloxacin & $0 \%$ \\
\hline Erythromycin & $0 \%$ \\
\hline Linezolid & $80 \%$ \\
\hline Teicoplanin & $80 \%$ \\
\hline Vancomycin & $60 \%$ \\
\hline Tetracycline & $60 \%$ \\
\hline Nitrofurantoin & $0 \%$ \\
\hline
\end{tabular}

Table 4. CAUTI and CA-ASB in various studies

\begin{tabular}{lccccl}
\multicolumn{1}{c}{ Study by } & Year & Total cases & CAUTI & CA-ASB & $\begin{array}{c}\text { Most common } \\
\text { organism } \\
\text { causing CAUTI }\end{array}$ \\
Bagchi et al. [10] & 2013 & 220 & $64(29 \%)$ & $156(71 \%)$ & E. coli \\
Kizilbash et al. [12] & 2013 & 308 & $128(42 \%)$ & $180(58 \%)$ & Candida sp. \\
Kaur et al. [4] & 2012 & 150 & $100(67 \%)$ & $50(33 \%)$ & E. coli \\
Bhatia et al. [11] & 2010 & 89 & $20(22 \%)$ & $69(78 \%)$ & E. coli \\
Taiwo and Aderounmu [14] & 2006 & 122 & $90(74 \%)$ & $14(26 \%)$ & Klebsiella sp. \\
Hazelett et al. [15] & 2004 & 57 & $34(60 \%)$ & $23(40 \%)$ & - \\
Tullu et.al. [16] & 1998 & 44 & $24(47 \%)$ & $10(20 \%)$ & E. coli \\
Garibaldi et al. [13] & 1974 & 405 & $95(23 \%)$ & $73(18 \%)$ & - \\
Present study & 2019 & 123 & $50(41 \%)$ & $73(59 \%)$ & E. coli \\
\hline
\end{tabular}

during their hospital admission. Hence, a closed catheter drainage system is suggested for short term and only to be used as and when needed $[8,9]$.

Pathogens directly enter the urinary tract through catheter or cystoscopy and cause infections. Bacteria can grow in either urine itself or on the surface of the catheter. After the attachment of bacteria on the catheter, it initiates biofilm formation. CAUTI may lead to secondary bacteremia which may damage the bladder $[8,9]$.
In the current study, we have compared the cases of CAUTI and CA-ASB recorded by various authors. Bagchi et al. [10], Bhatia et al. [11], and Kizilbash et al. [12] showed higher cases of CAASB and lower cases of CAUTI among catheterized patients. Similar data were found in our study also, while other studies showed more numbers of CAUTI against CA-ASB [4,13-16]. These differences in cases of CAUTI and CA-ASB are well expected as they are dependent on various infection control policies and catheter care of patients. A detailed surveillance for HAI including CAUTI is warranted for designing efficient and 
sustained infection control measures in all healthcare settings (Table 4).

In the present study, the most encountered uropathogen was $E$. coli, followed by Klebsiella sp. and Pseudomonas sp. in CAUTI patients. Other CAUTI studies also reported E. coli as the most common organism $[4,10,16]$. In case of CA-ASB, E. coli was reported as the most common pathogen, followed by Klebsiella sp. and Enterococcus sp. Similar findings were reported in a study by Bhatia et al. [11] (Table 4).

In this study the highest number of CAUTI were observed in the medicine ward $(40 \%)$, followed by the surgical ward $(32 \%)$. The isolation rate of CAUTI in ICUs was lower than the wards. These alterations are well expected as they depend on various local variables.

CAUTI isolates were found to be resistant to commonly used antibiotics like cephalosporins, fluoroquinolones, and sulfonamides. Similar findings were also reported by Kaur et al. [4], Bagchi et al. [10], and Taiwo and Aderounmu [14]. Higher antibiotics like Colistin, Tigecycline, and to some extent Imipenem were found to be effective against these pathogens. The present study showed $18 \%$ production of ESBL among CAUTI pathogens and $20 \%$ production of the MBL enzyme. This data is also supported by the study of Kaur et al. [4]. This alarming data of production of special resistance mechanisms and resistance to common drugs made the CAUTI more complex and difficult to treat.

The most common risk allied with nosocomial UTI is the urinary catheter. Many studies have shown that UTI incidences are increased with prolonged duration of catheter. Risk of infection is elevated by $1 \%-5 \%$ for each day of catheterization. After a single event of catheter insertion and its removal, $1 \%-20 \%$ of patients acquire CAUTI. When patients receive sterile closed collecting systems, the chances of CAUTI are $10 \%-25 \%$, while catheter draining into an open collecting vessel leads to $100 \%$ UTI of the patient within 4 days of hospitalization. Other risk factors like gender, impaired renal functions, and comorbidity also add up to the rate of nosocomial UTI. Systemic antibiotics may reduce the risk of UTI in patients $[8,9]$.

Different studies used different time frames for catheterization days. In the present study, we followed patients from more than 48 hours to a maximum 23 days of catheterization. Older age is also one of the important risk factors to cause CAUTI. The present study depicts a positive association between age and number of CAUTI cases. Cases were found to be more in the higher age groups. Maximum uropathogens were isolated from the more than 55 years age group. This data is also supported by Bagchi et al. [10] and Kaur et al. [4]. We have also studied the genderwise distribution of CAUTI isolates. Unlike community-acquired UTI where female patients are most commonly affected, here we have observed more cases of CAUTI in male patients $(68 \%)$ in comparison to female patients (32\%) (Table 1).

In the present study, we identified a number of predisposing factors responsible for CAUTI. One of the important factors of this list is type 2 diabetes mellitus. Diabetic patients are more prone to get CAUTI during the catheter days. This association is also supported by the study of Bagchi et al. [10]. Hence, patients with various predisposing factors warranted special catheter care during the hospital stay.

Monitoring of CAUTI incidences along with risk factors and predisposing factors is very important for treating physicians as causative microorganisms can be polyantimicrobial-resistant nosocomial pathogens [17]. Proper microbiological diagnosis is important for proper treatment [18]. Patients admitted to the hospital may have other co morbid conditions, immunosuppression, neutropenia, multi-organ involvement, and may have other hospital-acquired infections which may complicate the course of CAUTI $[19,20]$.

Prevention and successful treatment of CAUTI requires good microbiological service support and information with reporting of such incidents at various target locations of the hospital. The Center for Disease Control and Prevention (CDC) has developed the "Targeted Assessment for Prevention" strategy to prevent HAIs including CAUTI [21]. For successful treatment, correct antibiotic choice is important, an idea which can be obtained by proper urine culture method which is not only related to microbiology laboratory quality standards but also pre-laboratory procedures like collection, storage, and process of urine samples such that contamination with other microorganisms is avoided. As per CDC, this is part of the treatment and also includes the urine culture stewardship program of patients who are admitted in the hospital [22]. Antibiotic stewardship program is very important in relation to UTI in hospitalized patients as it is very frequently encountered by treating physicians [23]. CAUTI being the most common form of UTI in hospitalized patients; appropriate treatment, proper assessment of patient, laboratory investigations, urine culture sensitivity pattern, and proper justification of the use of particular antibiotic for proper duration becomes a part of antibiotic stewardship program at the local and global level [22,23]. Proper microbiological laboratory support, rational use of urinary catheters, catheter care, strict infection control practices, and appropriate antibiotic use are important for the prevention of CAUTI [19-24].

\section{CONCLUSION}

The present study showed a positive association between the number of CAUTI cases and various risk factors like age, catheter days, and disease like type 2 diabetes mellitus. Higher resistance of antibiotics along with special resistance mechanisms like MBL and ESBL made CAUTI more decisive. We have noted that CAUTI is more common in elderly patients, so special catheter care and precaution are warranted in the treatment of such patients.

\section{ACKNOWLEDGMENTS}

The authors would like to acknowledge with much appreciation the crucial role of Dr. J. D. Lakhani, Academic Director, Professor and Head, Department of Medicine, SBKS MI \& RC, for his vital suggestions in the planning and execution of this study.

\section{AUTHORS' CONTRIBUTION}

All the listed authors have made substantial, direct, and intellectual contributions to the work and have approved it for publication. 


\section{ETHICAL APPROVAL}

The study was approved by the institutional ethics committee (Human Research), C. U. Shah Medical College, Surendranagar, Gujarat, India. Ethical approval number: CUSMC/IEC(HR)/ APPROVAL/2017/4843. Date: 19/12/2017.

\section{LIST OF ABBREVIATIONS}

HAI: Hospital-acquired infection

ICU: Intensive care unit

UTI: Urinary tract infection

\section{CONFLICT OF INTEREST}

The authors report no financial or any other conflicts of interest in this work.

\section{FUNDING}

There is no funding to report.

\section{REFERENCES}

1. Thomas MH, Suzanne FB, Diana DC, Richard C, Suzanne EG, James CR, et al. Diagnosis, prevention, and treatment of catheter - associated urinary tract infection in adults: 2009 international clinical practice guidelines from the infectious disease society of America-IDSA. Clin Infect Dis 2010;50:625-63.

2. Turck M, Goffe B, Petersdorf RG. The urethral catheters and urinary tract infection. J Urol 1962 88:834-7.

3. Kass EH. Asymptomatic infections of the urinary tract. Trans Assoc Am Physicians 1956;69:56-63.

4. Kaur M, Date V, Lakhani SJ. Microbiological profile of catheter associated urinary tract infections in patients of Dhiraj hospital. Int J Curr Res 2017;9(08):55905-11.

5. Forbes BA, Sahm DF, Weissfeld AS. Bailey and Scott's diagnostic microbiology. 12th edition, Mosby Elsevier, Amsterdam, Netherlands, 2007.

6. Winn WJ, Allen S, Janda W, Koneman E, Procop G, Schreckenberger P, et al. Koneman's color atlas and textbook of diagnostic microbiology. 6th edition, Lippincott Williams and Wilkins Company, Philadelphia, PA, 2006.

7. Weinstein M. Performance standards for antimicrobial susceptibility testing. M100. 28th edition, Clinical and Laboratory Standards Institute, Wayne, PA, 2018

8. Neeta P, Satish P. Hospital associated infections: epidemiology, prevention and control. 2nd edition, Jaypee Brothers Medical Publisher, Chennai, India, 2017.

9. Sanjay S. Handbook of hospital infection control. 1st edition, CBS Publishers and Distributors, Delhi, India, 2016.

10. Bagchi I, Jaitly NK, Thombare VR. Microbiological evaluation of catheter associated urinary tract infection in a tertiary care hospital. Int J Biol Health Sci 2013;1(2):1-10.
11. Bhatia N, Daga MK, Garg S, Prakash SK. Urinary catheterization in medical wards. J Glob Infect Dis 2010;2(2):83-90.

12. Kizilbash QF, Petersen NJ, Chen GJ, Naik AD, Trautner BW. Bacteremia and mortality with urinary catheter-associated bacteriuria Infect Control Hosp Epidemiol 2013;34(11):1153-9.

13. Garibaldi RA, Burke JP, Dickman ML, Smith CB. Factors predisposing to bacteriuria during indwelling urethral catheterization. N Engl J Med 1974;291:215-9.

14. Taiwo SS, Aderounmu AOA. Catheter associated urinary tract infection: aetiologic agents and antimicrobial susceptibility pattern in Ladoke Akintola University Teaching Hospital, Osogbo, Nigeria. Afr J Biomed Res 2006;9(3):141-8.

15. Hazelett SE, Tsai M, Gareri M, Allen K. The association between indwelling urinary catheter use in the elderly and urinary tract infection in acute care. BMC Geriatr 2006;6(15):1471-2318.

16. Tullu MS, Deshmukh CT, Baveja SM. Urinary catheter related nosocomial infections in paediatric intensive care unit. J Postgrad Med 1998;44(2):35-9.

17. Kamat US, Fereirra A, Amonkar D, Motghare DD, Kulkarni MS Epidemiology of hospital acquired urinary tract infections in a medical college hospital in Goa. Indian J Urol 2009;25:76-80.

18. Wilson ML, Gaido L. Laboratory diagnosis of urinary tract infections in adult patients. Clin Infect Dis 2004;38(8):1150-8.

19. Jacobsen SM, Stickler DJ, Mobley HL, Shirtliff ME. Complicated catheter-associated urinary tract infections due to Escherichia coli and Proteus mirabilis. Clin Microbiol Rev 2008;21(1):26-59.

20. Sydnor ER, Perl TM. Hospital epidemiology and infection control in acute-care settings. Clin Microbiol Rev 2011;24:141-73.

21. www.cdc.gov. Atlanta: Targeted Assessment for Prevention (TAP) reports, 2019. Available via www.cdc.gov (Accessed 22 February 2021).

22. www.cdc.gov. Urine culture stewardship in hospitalized patients. CDC HealthCare Associated Infections, Atlanta, Georgia, 2019. Available via www.cdc.gov

23. Lakhani JD, Lakhani SJ, Meera S, Sanket P, Sandeep J. Appropriate use of antimicrobial agents in urinary tract infections: perception of physicians and resident doctors. J Integr Health Sci 2019;7:19-24.

24. Geneva: Guidelines on core components of infection prevention and control programmes at the national and acute health care facility level, 2016. Available via https:/www.who.int/infection-prevention/ publications/ipc-components-guidelines/en/ (Accessed November 2016)

\section{How to cite this article:}

Panjwani DM, Lakhani SJ, Mehta SJ, Kikani KM, Shah KS. A comprehensive study of microbiological profile, risk factors and antibiotic sensitivity pattern of catheter associated urinary tract infection in a teaching hospital of Gujarat. J Appl Biol Biotech 2021; 9(05):83-88. 